\title{
PARADOJAS DE LA RELACIÓN LOCAL-GLOBAL. ELEMENTOS PARA UNA TEORÍA CRÍTICA DE LA GLOBALIZACIÓN
}

\author{
Núria Benach Rovira *
}

Parece existir un consenso generalizado sobre la existencia de cambios rápidos en el mundo, fruto de la creciente velocidad de las comunicaciones, la interpenetración de la cultura y de la economía, el reconocimiento de la dimensión de los problemas ambientales, el crecimiento de las migraciones internacionales, el poder de los mercados financieros globales, etc. Un entramado complejo de cambios al que aludimos habitualmente bajo el nombre genérico de "globalización", y del que difícilmente puede alcanzarse ningún entendimiento sin tener en cuenta que un componente esencial para explicar los mencionados cambios es el espacio y la forma en que el capitalismo altera sus propriedades. Éste es, precisamente, el hilo conductor de este artículo en el que, tras una introducción a propósito del mismo de término de "globalización", necesaria para mostrar la dificultad casi insalvable que supone la confusión entre categoría de análisis y objeto de estudio, se abordan sucesivamente cuatro "paradojas" que no son sino cuatro posibles lecturas espaciales de la cuestión. En primer lugar, planteamos cómo puede ser nuevo algo tan antiguo, lo que remite tal vez al gran tema de las discontinuidades y de las inercias históricas y, sin duda, a la misma consideración de las fases por las que atraviesa el capitalismo. En segundo lugar, planteamos el mito de la creciente homogeneización (económica, cultural...) del mundo para revelar que, tras ese mito, se esconden justamente los mecanismos para una creciente diferenciación del espacio y de la sociedad. En tercer lugar, abordamos un tema cotidiano: la creciente capacidad de conexión, de desplazamiento y de conocimiento de lo que acontece en otras partes del mundo, con su consiguiente contrapeso: no todos estamos conectados, incluso puede que algunos estén más aislados de lo que han estado jamás. En cuarto lugar, siguiendo los términos de Manuel Castells, planteamos la dicotomía entre espacio de flujos y espacio de lugares para señalar que, una vez más, su relevancia analítica depende de a qué y de a quién nos estemos refiriendo. Finalizamos cambiando de plano (de escala conceptual) para señalar que los problemas de la dualidad global-local no son, en definitiva, más que los de la "tramposa cuestión" como la ha llamado D. Harvey (2000) de tener que decidir entre lo singular y lo general, entre lo homogéneo $y$ lo heterogéneo. No se trata, 
lógicamente, de tener que decidir entre dos opciones sino de encontrar una vía interpretativa que, sirviéndose de la diversidad de escalas como instrumento de análisis, logre superar los problemas de ilegibilidad del mundo actual (Jameson, F 1984) que son el resultado, en buena parte, de la "espacialidad diferencial" que señalara, hace años, Yves Lacoste (1977).

\section{A propósito del término globalización: ¿objeto o discurso?}

El término "globalización" se halla muy difundido en la actualidad y se ha venido utilizando ampliamente en los últimos 30 años hasta convertirse en el concepto hegemónico para aludir a la organización económica del capitalismo actual, utilizado, además, sin distinción de contenidos concretos, ideologías o intenciones. Es habitual relacionar el término "globalización" con el de "aldea global" que acuñara McLuhan en los años 60. Parece ser, no obstante, que un origen algo más específico puede encontrarse, significativamente, en la publicidad de American Express para señalar el alcance global de su tarjeta de crédito a mediados de los 70. El término se habría difundido ampliamente desde entonces en la prensa financiera y de negocios como una legitimación de la desregulación de los mercados financieros y a mediados de los 80 habría colaborado en la creación de una atmósfera de optimismo empresarial entorno a la liberalización de los mercados. Por ello, se ha señalado que no se trata en absoluto de un concepto inocente, sino que está íntimamente asociado al neoliberalismo económico imperante pese a que haya venido siendo utilizado también, de modo más bien acrítico, como descripción del estado del mundo sustituyendo otros conceptos más útiles y con más carga política como los de imperialismo o neocolonialismo (Harvey, D. 2000 ).

Desde hace varios decenios se utiliza, pues, el término de "globalización" como una manera sintética de designar este conjunto de cambios complejos relacionados con la llamada compresión espacio-temporal hasta llegar a convertirse en un objeto real más que en una simple categoría para el análisis (Allen, J. 1995). Y, como siempre, confundir el instrumento con el objeto de análisis no hace sino provocar grandes dificultades de analizar realmente a lo que se está aludiendo. Muchos autores se han lanzado a remarcar este hecho a propósito de la globalización. Es el caso de M. Crang (1999) que, utilizando categorías lefebvrianas, ha distinguido la globaliza-ción como "espacio concebido" del espacio percibido, - del mismo Milton Santos que se ha referido a la misma idea con la expresión de la "globalización como fábula" o "el mundo tal como nos lo hacen creer", contrapuesto al "mundo como perversidad" es decir, "el mundo como es" (Santos, M. 2000).

Pero fundir la representación con la realidad no es, naturalmente, una operación involuntaria ni inocua, sino que está siempre al servicio de la naturalización del conocimiento lo que, siguiendo a Roland Barthes, denominaríamos la creación de mitos, en este caso el "mito de la globalización" (Robertson, R. 1995). "Sin fábulas ni mitos, este periodo histórico no existiría tal como es", ha sentenciado M. Santos (Santos, M. 2000:43). Quizá N. Thrift lo haya señalado con acierto cuando afirma que ele problema de 
éstos $u$ otros discursos a propósito de la globalización no es lo que dicen sino, precisamente, lo que no dicen: que el mundo sea más difícil de entender no significa que sea ininteligible, que la velocidad de movimiento se haya acelerado no significa que sea lo único que exista, que el espacio esté conformado crecientemente por flujos, no significa que éstos lleguen a todas partes (Thrift, N. 1995).

\section{Globalización: ¿un fenómeno antiguo o nuevo?}

Quizá como consecuencia de la confusión entre categoría de análisis y objeto $y$, dado que el término es relativamente reciente, se tiende a acentuar lo novedoso de la globalización. Es bastante fácil dejarse llevar por el impacto de las nuevas tecnologías en todas las escalas y en todas las esferas, por la alteración de nuestro sentido de la distancia, por lo cualitativamente distinto. Pero, por otra parte, como diversos autores se han esforzado por señalar, la reorganización geográfica y la innovación técnica siempre han acompañado las salidas de la crisis del capitalismo $y$, en este sentido, la globalización habría existido desde hace mucho tiempo, por lo menos desde 1492. En una obra reciente, David Harvey ha recuperado de nuevo un clásico: la relectura del Manifiesto Comunista de Marx y Engels le permite establecer su tremenda actualidad para explicar los procesos actuales: el fetichismo de las mercancias que nos impide conocer las relaciones subyacentes a su producción, los escándalos de la explotación infantil, el cambio tecnológico y su impacto en el mercado de trabajo, el debilitamiento del trabajo organizado, la producción de un ejército de reserva industrial $^{l}$

La cuestión importante por la que nos hemos detenido en este tema de lo viejo/lo nuevo es que una lectura "continuista" como ésta conduce al reconocimiento de que las reestructuraciones geográficas y las estrategias espaciales son elementos vitales de la acumulación de capital, tanto hoy como históricamente: "Si el término globalización significa algo a propósito de nuestra geografía histórica reciente, es probable que sea una nueva fase de exactamente el mismo proceso que subyace a la producción capitalista del espacio" (Harvey, D. 2000:52). Desde luego que, desde Marx, han existido muchas otras formulaciones que, con diversos términos, han lanzados teorías a propósito de lo que hoy llamamos globalización, empezando por la misma teoría económica del imperialismo de Lenin de 1916. Sin embargo, las formulaciones contemporáneas han estado inspiradas grandemente por las propuestas de I. Wallerstein a propósito de la génesis del moderno sistema mundial $y$ que, juntamente con otras versiones de la acumulación a escala mundial como la teoría de la dependencia, el intercambio desigual o la teoría de la nueva división internacional del trabajo forman el cuerpo teórico que ha fundamentado una gran parte de las aproximaciones existentes.

Puede afirmarse, pues, que existe un notable consenso en la afirmación de que lo que hoy llamamos globalización habría existido, de hecho, durante mucho tiempo. Algunos autores, sin embargo, se han esforzado por remarcar las novedades que han surgido en las últimas décadas. Peter Dicken, por ejemplo, alude a lo pasado como "internacionalizacion" para señalar 
que, en la actualidad, la actividad económica no estaría sólo más internacionalizada, sino también más globalizada. Internacionalización y globalización no son, pues, para este autor, términos sinónimos, sino que el primero alude meramente a un creciente ámbito geográfico de las actividades económicas a través de las fronteras nacionales mientras que la globalización, cualitativamente diferente, implicaría un grado de integración funcional entre actividades económicas dispersas internacionalmente. (Dicken, $P$. 1992:1).

Aún insistiendo en todo momento que la globalización no es en absoluto un fenómeno nuevo, Doreen Massey ha señalado, por su parte, cómo un trazo novedoso puede residir en la nueva dirección que han tomado algunos flujos (particularmente, las migraciones) que habría supuesto la "llegada de los márgenes al centro" $y$, sobre todo, en la existencia de un mayor estímulo para el surgimiento de cuestiones conceptuales, especialmente, a propósito de xla naturaleza de los lugares y de una creciente sensibilidad hacia lo diferente (Massey, D. y Jess, P. 1995).

\section{3. ¿Un mundo más homogéneo o más diferenciado?}

La búsqueda de una interpretación a los grandes cambios a los que está sometido el mundo actual habría dado lugar a dos respuestas analíticas (Cochrane, A. 1995). En primer lugar, la que enfatiza los aspectos globales del cambio, según la cual ningún caso particular puede entenderse lejos de la dinámica global que lo determina. Es una idea que sugiere la existencia de un proceso de homoge- neización social y cultural. En sus versiones más extremas, se ha llegado a proclamar, del mismo modo que Fukuyama lo hiciera años atrás a propósito de la historia, el "fin de la geografía" (Virilio, P. 1997) y se ha puesto el acento en cómo la actividad humana trasciende fronteras $y$ "se libera de sus cadenas geográficas" (Lasserre, F., 2000). Pero incluso en interpretaciones más mesuradas, las que enfatizan la importancia de los procesos globales sin que por ello se tenga que afirmar que la globalización significa lo mismo en todas partes, se parte de la idea de que lo global determina, o por lo menos afecta, el devenir de lo local. El supuesto subyacente a este tipo de aproximaciones es que los factores globales son dinámicos mientras que los locales son necesariamente conservadores, fuentes de resistencia más que bases para el cambio.

Por otro lado, una segunda vía interpretativa, más acorde con los dictados postmodernos, pone el énfasis en la fragmentación y la localización de la experiencia vivida. En lugar de homogeneización, el acento se pone en la diversidad, en los modos en los que incluso los iconos culturales dominantes son interpretados localmente y en los significados que adquieren en diferentes sitios. Por ejemplo, en un manual reciente de Geografía cultural se aporta, en la primera página, una "prueba" de por qué la globalización no conduce a la homogeneización, sino, en este caso, a la diferenciación cultural incluso tratándose de uno de los máximos símbolos de la presión globalizadora: la adaptación de los menús de McDonald's a los gustos de las culturas locales; así, uno puede pedir cerveza y vino en Europa, burgers de teriyaki en Japón, y arroz en China (Mitchell, D. 2000) 
Desde esta perspectiva, se apreciaría una sensible alteración de las pautas centralizadas del pasado que haría prácticamente imposible aprehender el mundo como una unidad. Muestra de ello sería, a nivel de la organización económica, cómo las grandes empresas están siendo forzadas a reorganizarse descentralizándose, franquiciándose o fragmentándose; a nivel cultural, en la vitalidad que muestran las culturas locales; en lo político, en el debilitamiento del estado y el afianzamiento de los regionalismos $y$ nacionalismos infraestatales y el crecimiento de redes más complejas de base local que tienen a puentear las jerarquías tradicionales.

En los extremos, ambas interpretaciones son, naturalmente, parciales ("metáforas de la realidad" los han llamado M. Santos y M.L. Silveira) y tienden a sobreenfatizar un aspecto en detrimento de otros: la globalización no significa el fin de la diferencia, ni tampoco el reconocimiento de la diferenciación debe significar que las fuerzas globales sean irrelevantes. Bien al contrario, es precisamente la interconexión entre estos procesos lo que define "la condición de la postmodernidad" No se trata de combinar los dos polos como si la respuesta correcta se hallara en algún punto medio, sino de mostrar que la creciente interdependencia entre lugares y actividades significa que las escalas geográficas han devenido más difíciles de separar. Como se ha señalado, la globalización es, antes que nada, una fuente de desarrollo desigual y, por tanto, de unicidad de los lugares, que, "como más se mundializan, más únicos se vuelven" (Santos, M. 1996).

\section{La "compresión espacio-temporal", ¿conecta o aisla?}

Las nuevas tecnologías del transporte $y$ de la comunicación han alterado profundamente la relación espacio-tiempo y han reducido de modo drástico el significado de las distancias hasta hacer común la metáfora de que "el mundo se encoge" Es lo que Janelle ha venido denominando ya desde 1969 "convergencia espacio-tiempo" (Janelle, D. 1991) para describir la reducción de las distancias en términos temporales o lo que Harvey ha llamado "compresión espacio-temporal" para referirse más específicamente a la expansión de las relaciones capitalistas de producción a una escala global (Harvey, D. 1989). El efecto de dicho cambio de la relación espaciotiempo es bien perceptible en nuestra vida cotidiana $y$ en nuestras concepciones cotidianas del espacio y del tiempo: las nuevas tecnologias permiten que seamos más móviles y que tengamos acceso a más información. Dicho de otro modo, el mundo se encoge no sólo porque sea más fácil y más barato viajar sino porque tenemos, gracias a las imágenes visuales generadas por los medios de comunicación, una idea del mundo sin tener que desplazarnos gracias a las representaciones del mundo que proporciona la televisión en sus informativos, series de ficción, documentales...incluso de los conflictos bélicos tal como la cobertura informativa en directo de la guerra del golfo de 1991 se encargó de demostrar (Taylor, P 1992). Sin embargo, hay que profundizar algo más en este argumento para comprobar si, efectivamente, potencial de movimiento significa posibilidad real de desplazamiento y si acceso a mu- 
cha más información significa estar, efectivamente, más informado (dado que la sobreexposición conduce casi con toda seguridad a la confusión). Es evidente que ni todo el mundo tienen el mismo acceso a las tecnologias ni se beneficia del mismo modo. Se ha señalado incluso que el énfasis en una compresión espacio-temporal que, ni es igual para todo el mundo ni para todas las esferas de actividad, no es sino una típica visión etnocéntrica occidental (Massey, D. 1993). Ello no significaría, sin embargo, que no todos estuviéramos recibiendo, de un modo u otro, su impacto pero los diferentes grupos sociales, los diferentes individuos se hallan colocados de modo muy diverso en estos flujos y conexiones. Como ha señalado Massey, la mayor parte de los que llevan a cabo el movimiento son los que lo controlan (distribuidores de películas, agencias de noticias, los que organizan inversiones $y$ transacciones a nivel internacional), en los márgenes están los que hablan de ello (académicos y periodistas), más allá, personas que se mueven físicamente sin estar encargados del proceso (refugiados, migrantes, sin papeles) o los que reciben las consecuencias de ello sin siquiera salir de casa. Y, por otro lado, la compresión espacio-temporal no sólo facilita el movimiento y la comunicación, también puede llegar a aislar a aquellos que no tienen acceso. Mike Davis ha descrito cómo en un centro neurálgico de las comunicaciones mundiales, Los Angeles, se puede formar un auténtico "guetto electrónico", en el que ni el espacio de flujos ni la compresión espacio-temporal alcanzan a tener ningún significado (Thrift, N. 1995). Manuel Castells ha utilizado la misma idea a otra escala para referirse al "apartheid tecnológico" del continente africano, excluido de la revolución tecnológica de la información a menudo no por falta de ordenadores, sino de electricidad. Lo más curioso es, sin embargo, que incluso cuando ciertos territorios llegan a estar conectados lo están sólo a efectos de su conexión mundial: "en 1996, 22 capitales africanas tenían plena conexión con Internet pero solo en un país -Senegalera posible el acceso fuera de la capital (Castells, M. 1998:117-119). Más aún, la movilidad de unos grupos puede provocar el aislamiento de otros. La movilidad del capital frente a la relativa inmovilidad de la fuerza de trabajo es una fuente constante de ejemplos a propósito de cómo el trabajo ganado en un territorio es a costa siempre del perdido en otro ${ }^{2}$ Ejemplos cotidianos como el uso del coche que, aumentando la movilidad de quien lo utiliza al tiempo que reduce la del que no pose automóvil al incidir en la viabilidad financiera del sistema de transporte público (y lo mismo se podría afirmar de los teléfonos móviles frente a las cabinas telefónicas, o de la telecompra frente al supermercado, etc.), no hacen sino aportar argumentos en favor de abandonar la idea de una compresión espacio-temporal generalizada e indiferenciada.

Así que la conclusión a la que llegamos es que la compresión espaciotemporal simultáneamente conecta y aisla pero afectando a diferentes territorios y a diferentes personas. Aún cabe añadir una nueva vuelta de tuerca al argumento para señalar que las nuevas tecnologías no sólo han intensificado la difusión de la información sino que han permitido también algunos cambios cualitativos; la globalización facilita también los instrumentos para su crítica tal como muestra el uso de Internet por parte de 
movimientos de resistencia a la globalización en sus muy diversas formas: desde el movimiento zapatista a las campañas anti-globalización, pasando por movimientos ecologistas diversos. Tal vez ello deba leerse como una de las características de la presente fase de globalización, esto es, como una fase marcada por la emergencia (o tal vez cabría decir por una mayor visibilidad) de la diferencia.

\section{Global o local: ¿Espacio de flujos o espacio de lugares?}

Hemos escogido la terminología de Castells de contraposición entre espacio de flujos o espacio de lugares como símbolo de la presentación antitética entre la escala global y la escala local. Se tratará de demostrar, finalmente, que no se trata de escalas competitivas sino de dos caras de la misma moneda. Para ello, puede ser útil el esquema que aporta crang a propósito de las tres grandes interpretaciones de la relación local-global (Crang, P 1999):

a) el mundo como mosaico o la consideración de los lugares como piezas de un conjunto (la visión de sentido común, la que se presenta a si misma como una realidad obvia). El énfasis se pone aquí en las fronteras de las áreas, ya que cada área tiene unas características distintivas que son las que permiten comprenderla. Cualquier intrusión en un área distintiva tiende a ser vista como una amenaza a su carácter Esta visión, que tiene el mérito de reconocer la diferencia, tiende, sin embargo, a fosilizarla y a utilizaria como parte de un localismo defensivo que legitima la exclusión social y espacial. b) el mundo como sistema. Las diferencias entre lugares son contempladas aquí como el producto de un sistema global. Así, éstas no serían fruto de sus cualidades internas sino de su localización en un contexto más amplio $y$ de las relaciones globales entre lugares.

c) el mundo como red. En esta visión, lo local y lo global no son contemplados como escalas diferentes sino como dos maneras de aproximarse a las mismas redes sociales y espaciales. Redes en las que lo local es global, y lo global es local.

Esta última interpretación trae a colación el término de "glocalización" como en el caso del término "globalización" se trata de una expresión muy utilizada en el mundo de la empresa. Según Robertson, fue una de las palabras de mayor éxito en el marketing en los años 90, utilizada para crear y publicitar mercancías y servicios sobre una base global pensando en mercados particulares crecientemente diferenciados (Robertson, R. 1995). El "micromarketing" persigue precisamente la construcción de consumidores cada vez más diferenciados sobre la base de que "la diversidad vende" (una idea, en definitiva, muy cercana a la de la distinción como base para la formación de capital cultural de Bourdieu). En el ámbito académico, sin embargo, el término se ha utilizado para romper la polaridad entre los términos global y local, donde lo local sólo puede ser una forma de oposición o de resistencia a lo hegemóni-camente global. Se trata, pues, de afirmar que lo local no es un mero contrapunto de lo global sino que es un aspecto fundamental de la globalización, lo que implica tener plenamente presente la simultaneidad e interpenatracion de lo que llamamos 
local y global. Un ejemplo puede ilustrar esta idea. Stuart Hall, a propósito de la taza de té como símbolo supremo de la identidad británica ha señalado cómo, en primer lugar, no existiendo ni una sola plantación de té en el Reino Unido, el té "inglés" proviene, naturalmente, de Ceilán o de la India, lo que remite a la misma formación de la identidad británica en su historia imperial. Añade: "como yo, muchos llegaron a Inglaterra en los 50 desde las Antillas, así que yo debo ser el azúcar de la taza de té inglés. Y muchos otros en el mundo son... la taza misma" (citado por P. Crang, 1999). La identidad británica no puede desvincularse de su pasado imperial de modo que ni siquiera "lo inglés" puede considerarse simplemente inglés sino que sólo puede comprenderse como parte de una red global.

Así que la cuestión que surge en este punto es la que formulaba Doreen Massey a propósito del papel de lo local: ¿qué sentido puede tener el lugar en este momento de creciente globalización? $\mathrm{O}$, dicho de otro modo, cómo se puede mantener una noción de diferencia geográfica, de unicidad, sin caer en las reacciones defensivas para la preservación de la identidad que han tomado frecuentemente formas reaccionarias como ciertas de formas de nacionalismo, la superprotección de patrimonios, xenofobias, etc. A partir de esta reflexión, Massey ha postulado la necesidad de reivindicar el "sentido del lugar", pero, eso si, un "sentido global del lugar" que parta de la consideración de que lo que proporciona la especificidad a un lugar no es una historia internalizada sino el hecho de estar construida a partir de un conjunto de relaciones con otros espacios (Massey, D. 1993).

\section{Problemas de escala: ¿problemas metodológicos o de objeto de estudio?}

Lo visto anteriormente puede llevar a pensar que, efectivamente, nos hallamos ante diversas paradojas contenidas en el examen de la relación local-global. Así, para sintetizar las cuatro paradojas que hemos explorado:

1. Lo global es antiguo, pero si algo cualitativamente nuevo tiene es que es más local.

2. En ciertos sentidos el mundo es más homogéneo pero en otros aspectos es más diferente. Y la misma globali-zación que homogeneiza el mundo es la responsable de su creciente desigualdad.

3. La llamada compresión espacio-temporal aumenta la conectividad pero también aisla personas y espa-cios. De hecho, es el aumento de la conectividad el que provoca el creciente aislamiento

4. El mundo es, crecientemente, un espacio de flujos. Y como más intensos esos flujos, mayor relevancia adqui-eren los lugares.

Llegados a este punto, quizá se imponga una reflexión de tipo metodológico. Lo global y lo local, lo singular y lo general, lo homogéneo y lo diferente, no son sino maneras distintas de contemplar las mismas realidades. Como muchos autores se han encargado de destacar, no existen escalas predefinidas como categorías ontológicas intocables, sino que la escala -tanto si se refiere a la extensión espacial de un fenómeno 
como si se refiere al nivel en el que los procesos son relevantes- es, siem-pre, una construcción social (Marston, S. 2000). Hay que decir, pues, que cuando tratamos lo global y lo local, no nos enfrentamos a objetos de estudio diferentes (no hay objetos locales y otro globales), sino que la diferencia está sólo en dónde se pone el énfasis y que para decidir, hay que prestar atención a la escala en la que los procesos analizados son relevantes 0 explicables. Pero, aún más, hay que destacar, y éste es el objetivo final de este artículo, que no es posible entender lo local sin lo global, ni lo global sin lo local. O, dicho de otro modo, que hay que globalizar lo local y localizar lo global. Porque no se trata de reivindicar, sin más, una vuelta a lo local, no hay que temer un retorno al empiricismo (Smith, N. 1987), sino que de lo que se trata es de buscar la causalidad de los cambios globales en el nivel local, que es, a su vez, producto de su posición con relación a fuerzas más amplias. Muchos autores se han detenido,

\section{Notas}

1. Las "coincidencias" son, ciertamente, impresionantes en el fragmento que sigue al famoso "todo lo sólido se desvanece en el aire": Espoleada por la necesidad de dar cada vez mayor salida a sus productos, la burguesía recorre el mundo entero. Necesita anidar en todas partes, establecerse en todas partes, crear vínculos en todas partes. Mediante la explotación del mercado mundial, la burguesía dio un carácter cosmopolita a la producción y al consumo de todos los paises. Con gran sentimiento de los reaccionarios, ha quitado a la industria su base nacional. Las antiguas industrias nacionales han sido destruidas y están destruyéndose continuamente. Son suplantadas por nuevas industrias, cuya introducción se convierte en cuestión vital para todas las naciones civilizadas, por industrias lógicamente, en esta cuestión crucial: Thrift ha señalado, a propósito de las teorías de Jameson, Harvey o Castells, que hay que ser más cuidadoso y no caer en exageraciones gratuitas (Thrift, N., 1995). Allen y Thompson, de su lado, han remarcado la necesidad de que hablar de globalización significa siempre necesariamente hablar también de un contexto concreto (Allen, J. y Thompson, G., 1997). Y si la relación entre lo global y lo local debe entenderse necesariamente de una forma dialéctica para ser explicativa, lo mismo puede afirmarse a propósito de las dicotomias singular-general o homogéneodiferente que dominan los debates teóricos de la Geografía Humana actual. Podríamos concluir, con Linda McDowell, que hay que continuar hablando de las grandes cuestiones pero sin menospreciar por ello el significado de los lugares y de las personas (McDowell, L., 1997). Ahi reside seguramente la clave para la construcción de una teoría crítica de la globalización.

que ya no emplean materias primas indigenas, sino materias primas venidas de las más lejanas regiones del mundo, y cuyos productos no sólo se consumen en el propio país, sino en todas las partes del globo. En lugar de las antiguas necesidades, satisfechas con productos nacionales, surgen necesidades nuevas, que reclaman para su satisfacción productos de los países más apartados y de los climas más diversos. En lugar del antiguo aislamiento de las regiones y naciones que se bastaban a si mismas, se establece un intercambio universal, una interdependencia universal de las naciones. $Y$ esto se refiere tanto a la producción material, como a la producción intelectual (...) Merced al rápido perfeccionamiento de los instrumentos de producción y al constante progreso de los 
medios de comunicación, la burguesía arrastra a la corriente de la civilización a todas las naciones, hasta a las más bárbaras. Los bajos precios de sus mercancias constituyen la artillería pesada que derrumba todas las murallas de China y hace capitular a los bárbaros más fanáticamente hostiles a los extranjeros. Obliga a todas las naciones, si no quieren sucumbir, a adoptar el modo burgués de producción, las constriñe a introducir la llamada civilización, es decir, a hacerse burgueses. En una palabra: se forja un mundo a su imagen y semejanza (Marx, K., Engels, F 1975 , orig. 1848).

2. Por ejemplo, leemos en La Vanguardia del 7 de mayo del 2001 bajo el titular de "Las multinacionales se van al Este" como diversas multinacionales dejan de producir en sus plantas occidentales para beneficiarse de las ventajas fiscales y de mano de obra de sus plantas en Europa oriental ("La japonesa Sony ha anunciado que dejará de producir 700.000 teleisores de gama baja en Viladecavalls, en favor de la planta qie tiene en la localidad eslovaca de Transua")

\section{Bibliografia}

ALLEN, J. "Global worlds" en Geographical Worlds, (Eds. ALLEN, J. y MASSEY, D.), The Open University, Milton Keynes, 1995.

ALLEN, J. y HAMNETT, C. (Eds.) A Shrinking World? Global Unevenness and Inequality, The Open University, Milton Keynes, 1995.

ALLEN, J. y THOMPSON, G. "Think global, then think again -economic globalization in context", Area, 29, 1997

BORJA, J. y CASTELLS, M. Local y Global. La gestión de las ciudades en la era de la información, Taurus, Madrid, 1998.

BRUNN, S. D. y LEINBACH, T R. (Eds.) Collapsing Space \& Time. Geographic Aspects of Communication \& Information, HarperCollins Academic, London, 1991.

CASTELLS, M. The Informational City, Basil Blackwell, Cambridge, Mass, 1991.

CASTELLS, M. La era de la información. Economía, sociedad y cultura. vol. 1: La sociedad-red, Alianza Editorial, Madrid, 1998.
CASTELLS, M. La era de la información. Economía, sociedad y cultura. vol. 3: Fin de milenio, Alianza Editorial, Madrid, 1998. COCHRANE, A. "Global worlds and worlds of difference" en A Global World? Reordering Political Space, (Eds. ANDERSON, J., et al.), The Open University, Milton Keynes, 1995.

COOKE, P. Back to the future: modernity, postmodernity and locality, Unwin Hyman, London, 1989.

COX, K. (Ed.) Spaces of Globalization: Reasserting the Power of Local, Guilford Press, New York, 1997

CRANG, M. "Globalization as Conceived, Perceived and Lived Spaces", Theory, Culture \& Society, 16, 1999.

CRANG, P. "Local-global" en Introducing Human Geographies, (Eds. CLOKE, P., et al.), Arnold, London, 1999.

DICKEN, P. Global shift, Harper \& Row, London, 1992.

DOLLFUS, O. La mundialización, Edicions Bellaterra, Barcelona, 1999. 
DUNCAN, S. "What is locality?" en New Models in Geography, Vol. 2 (Eds. PEET, R. y THRIFT, N.), Unwin Hyman, London, 1989.

FEATHERSTONE, M. Global culture, Sage, London, 1990.

FEATHERSTONE, M., et al. (Eds.) Global Modernities, Sage, London, 1995.

GIDDENS, A. Consecuencias de la modernidad, Alianza Universidad, Madrid, 1993.

HARVEY, D. The Condition of Postmodernity, Basil Blackwell, Oxford,1989.

HARVEY, D. Spaces of hope, Edinburgh University Press, Edinburgh, 2000.

HIRST, P y THOMPSON, G. Globalization in question, Polity Press, Cambridge, 1996.

JAMESON, F. "Postmodernism, or the Cultural Logical of Late Capitalism", New Left Review, 1984.

JANELLE, D. "Global interdependence and its consequences" en Collapsing Space \& Time. Geographic Aspects of Communication \& Information, (Eds. BRUNN, S. D. y LEINBACH, T R.), Harper Collins Academic, London, 1991.

JOHNSTON, R. J., et al. (Eds.) Geographies of global change, Blackwell, London, 1995.

KELLNER, D. (2000) "Globalization and the Postmodern Turn" (http://www.gseis. ucla.edu/courses/ed253a/dk/globpm.htm) KNOX, P.L. and S.A. MARSTON, Human Geography. Places and Regions in Global Context. 1998, Upper Saddle River, N.J.: Prentice Hall, 1989.

LACOSTE, Y La Geografía: un arma para la guerra, Anagrama, Barcelona, 1977
LASSERRE, F (2000) "Internet : La fin de la géographie ?" Cybergeo, http://193.55. 107.3/ptchaud/lasser.htm\#note4.

MARSTON, S. A."The social construction of scale", Progress in Human Geography, 24, 2000.

MARX, K. y ENGELS, F (1975) Obras escogidas, Akal, Madrid.

MASSEY, D. "Power-geometry and a progressive sense of place" en Mapping the futures: local cultures, global change, (Eds. BIRD, J., et al.), Routledge, London, 1993.

MASSEY, D. "A Global Sense of Place" en Exploring Human Geography. A Reader, (Eds. DANIELS, S. y LEE, R.), Arnold, London, 1996.

MASSEY, D., L'"Estudi de localitats" en Geografia Regional. Treballs de la Societat Catalana de Geografia, 1990.

MCDOWELL, L. "Understanding Diversity: The Problem of/for "theory"" en Reading Human Geography. The Poetics and Politics of Inquiry, (Eds. BARNES, $\mathrm{T} y$ GREGORY, D.), Arnold, London, 1997

MEEGAN, R. "Local worlds" en Geographical Worlds, (Eds. ALLEN, J. y MASSEY, D.), The Open University, Milton Keynes, 1995.

MEYER, W. B., et al. "The Local-Global Continuum" en Geography's Inner Worlds, (Eds. ABLER, R., et al.), Rutgers, Rutgers, N.J., 1992.

MITCHELL, D. Cultural Geography A Critical Introduction, Blackwell, Oxford, 1997

NEDERVEEN PIETERSE, J. "Globalization North and South. Representations of Uneven Development and the Interaction of Modernities", Theory, Culture \& Society, $17,2000$. 
PEET, R. "World capitalism and the destruction of regional cultures" en The world in crisis, (Eds. JOHNSTON, R. J. y TAYLOR, P ), Blackwell, Oxford, 1989.

RELPH, E. Place and placelessness, Pion, London, 1976.

ROBINS, K. "The New Spaces of Global Media" en Geographies of Global Change. Remapping the World in the Late,. Twentieth Century, (Eds. JOHNSTON, R. J., et al.), Blackwell, Oxford, 1992.

SANTOS, M. De la totalidad al lugar, Oikos-Tau, Vilassar de Mar, 1996.

SANTOS, M. y SILVEIRA, M. L. "Más allá de las metáforas...Una geografía de la globalización", Estudios Geográficos, (LVIV)230, 1998

SANTOS, M. Por uma outra globalização: do pensamento unico à consciência universal, Record, Rio de Janeiro, 2000.

SMITH, N. "Dangers of the empirical turn", Antipode, 1987

SMITH, N. (1993) "Homeless/global: scaling places" en Mapping the futures: local cultures, global change, (Eds. BIRD J., et al.), Routledge, London, pp. 87-119.

SOJA, E. Postmodern Geographies, Verso, London-New York, 1989.

SWYNGEDOUW, $E$. "The heart of the place: the resurrection of locality in an age of hyperspace", Geografisker Annaler, 71B, 1989.

TAYLOR, P. War and the Media: Propaganda and Persuasion in the Gulf War, Manchester University Press, Manchester, 1992.

THRIFT, N. "A Hyperactive World" en Geographies of Global Change. Remapping the World in the Late Twentieth Century, (Eds. JOHNSTON, R. J., et al.), Blackwell, Oxford, 1995.

VELTZ, P Mundialización, ciudades y territorios: la economía de archipiélago, Ariel, Barcelona, 1999.

VIRILIO, P. "Fin de I'histoire, ou fin de la géographie? Un monde surexposé", Le Monde Diplomatique, agosto 1997

Concluido em abril de 2002. 